\title{
Modellbasierte Beschreibung von Umfangskräften bei kleinen Geschwindigkeiten
}

Paul Heimann, AUDI AG

This manuscript is not available according to publishing restriction. Thank you for your understanding. 